\title{
New island of cluster emitters
}

\author{
D. N. Poenaru* and W. Greiner \\ Gesellschaft für Schwerionenforschung, Postfach 110552, D-6100 Darmstadt, Germany \\ and Institut für Theoretische Physik der J. W. Goethe Universität, Postfach 111932, D-6000 Frankfurt am Main, Germany \\ R. Gherghescu \\ Institute of Atomic Physics, P.O. Box MG-6, RO-76900 Bucharest, Romania
}

(Received 9 July 1992)

\begin{abstract}
A new region of proton-rich parent nuclei decaying by spontaneous cluster emission with a measurable branching ratio relative to alpha decay is predicted within the analytical superasymmetric fission model. After a brief presentation of the model and of the seven mass tables used to calculate the released energy, the obtained results are discussed. Measurable half-lives and branching ratios are estimated for ${ }^{12} \mathrm{C},{ }^{16} \mathrm{O},{ }^{28} \mathrm{Si}$, and other cluster radioactivities of some nuclides having proton and neutron numbers in the range $Z=56-64$ and $N=58-72$. Such nuclei far from stability could be produced in reactions induced by radioactive beams.
\end{abstract}

PACS number(s): 23.70. $+\mathrm{j}, 27.60 .+\mathrm{j}$

\section{INTRODUCTION}

New kinds of natural charged-particle radioactivities, in which the emitted cluster is heavier than an $\alpha$ particle but lighter than a fission fragment, were predicted in 1980 , when it was shown that ${ }^{222} \mathrm{Ra}$ and ${ }^{224} \mathrm{Ra}$ should emit with maximum probability ${ }^{14} \mathrm{C}$ (see the review papers $[1,2]$ and the references therein). The first version of our analytical superasymmetric fission model (ASAFM) were developed in the same year, and it has been frequently exploited to estimate the half-lives used to guide the experiments. The theory was improved successively to give better account of the measured effects. Other fission- and cluster-preformed models were published after 1985 (see the review [3]).

Several successful experiments have been performed since 1984 , when Rose and Jones discovered ${ }^{14} \mathrm{C}$ emission from ${ }^{223} \mathrm{Ra}$. The highest branching ratio relative to $\alpha$ decay from the whole region of parents leaving a daughter around ${ }^{208} \mathrm{~Pb}$ was measured on this occasion. From more than 140 different kinds of new decay modes predicted within the ASAFM, the following cluster radioactivities are already confirmed: ${ }^{14} \mathrm{C},{ }^{20} \mathrm{O},{ }^{24,25} \mathrm{Ne}$, ${ }^{28,30} \mathrm{Mg}$, and ${ }^{32,34} \mathrm{Si}$ (see the review papers $[4,5]$ and also the Refs. [6, 7]). They have been detected in the region of trans-radium parent nuclei (more precisely in the nuclides ${ }^{222-224,226} \mathrm{Ra},{ }^{225} \mathrm{Ac},{ }^{228,230} \mathrm{Th},{ }^{231} \mathrm{~Pa},{ }^{232-234} \mathrm{U}$, ${ }^{236,238} \mathrm{Pu}$, and ${ }^{242} \mathrm{Cm}$ ).

A new edition of our extended tables has recently been published [8], updated according to the latest improvements of the model and extended into regions of nuclei far from stability and superheavies.

\footnotetext{
*Permanent address: Institute of Atomic Physics, P.O. Box MG-6, RO-76900 Bucharest, Romania.
}

In the following we shall focus our attention on a relatively small island of proton-rich parents, which could be produced in the near future in reactions induced by radioactive beams. The main interest for this island comes from the estimated favorable branching ratios. The residual daughter nuclei are grouped around ${ }^{100} \mathrm{Sn}$, as in case of the island of $\alpha$-particle emitters measured at Gesellschaft für Schwerionenforschung (GSI) Darmstadt many years ago [9].

\section{MODEL}

The half-life of a parent nucleus $(A, Z)$ against the split into a cluster $\left(A_{e}, Z_{e}\right)$ and a daughter $\left(A_{d}, Z_{d}\right)$ is calculated by using the Wentzel-Kramers-Brillouin (WKB) semiclassical approximation

$$
\begin{aligned}
& T=\left[(h \ln 2) /\left(2 E_{v}\right)\right] \exp \left(K_{\mathrm{ov}}+K_{s}\right), \\
& K=K_{\mathrm{ov}}+K_{s}=\frac{2}{\hbar} \int_{R_{a}}^{R_{b}}\left(2 \mu\left\{\left[E(R)-E_{\mathrm{cor}}\right]-Q\right\}\right)^{1 / 2} d R,
\end{aligned}
$$

where $\mu=m A_{e} A_{d} / A$ is the reduced mass, $m$ is the nucleon mass, and $E(R)$ is the interaction energy of the two fragments separated by the distance $R$ between centers. $E_{\text {cor }}$ is a correction energy similar to the Strutinsky shell correction, also taking into account the fact that the Myers-Swiatecki liquid drop model (LDM) overestimates fission barrier heights and the effective inertia in the overlapping region is different from the reduced mass [10]. As usual, $h$ is the Planck constant and $E_{v}$ is the zero point vibration energy. The turning points of the WKB integral are

$$
R_{a}=R_{i}+\left(R_{t}-R_{i}\right)\left[\left(E_{v}+E^{*}\right) / E_{b}^{0}\right]^{1 / 2},
$$




$$
R_{b}=R_{t} E_{c} \frac{\frac{1}{2}+\left[\frac{1}{4}+\left(Q+E_{v}+E^{*}\right) E_{l} / E_{c}^{2}\right]^{1 / 2}}{\left(Q+E_{v}+E^{*}\right)}
$$

where $E^{*}$ is the excitation energy concentrated in the separation degree of freedom, $R_{i}=R_{0}-R_{e}$ is the initial separation distance, $R_{t}=R_{e}+R_{d}$ is the touching point separation distance, $R_{j}=r_{0} A_{j}^{1 / 3}\left(j=0, e, d ; r_{0}=1.2249\right.$ $\mathrm{fm})$ are the radii of parent, emitted, and daughter nuclei, and $E_{b}^{0}=E_{i}-Q$ is the barrier height before correction.
The interaction energy at the top, in the presence of a non-negligible angular momentum $l \hbar$, is given by

$$
E_{i}=E_{c}+E_{l}=e^{2} Z_{e} Z_{d} / R_{t}+\hbar^{2} l(l+1) /\left(2 \mu R_{t}^{2}\right) .
$$

The two terms of the action integral $K$, corresponding to the overlapping $\left(K_{\text {ov }}\right)$ and separated $\left(K_{s}\right)$ fragments, are calculated by the analytical formulas (approximated for $K_{\mathrm{ov}}$ and exact for $K_{s}$ in case of separated spherical shapes within the LDM)

$$
\begin{aligned}
K_{\mathrm{ov}}= & 0.2196\left(E_{b}^{0} A_{e} A_{d} / A\right)^{1 / 2}\left(R_{t}-R_{i}\right)\left[\sqrt{1-b^{2}}-b^{2} \ln \frac{1+\sqrt{1-b^{2}}}{b}\right], \\
K_{s}= & 0.4392\left[\left(Q+E_{v}+E^{*}\right) A_{e} A_{d} / A\right]^{1 / 2} R_{b} J_{r c}, b^{2}=\left(E_{v}+E^{*}\right) / E_{b}^{0}, \\
J_{r c}= & (c) \arccos \sqrt{(1-c+r) /(2-c)}-[(1-r)(1-c+r)]^{1 / 2} \\
& +\sqrt{1-c} \ln \left[\frac{2 \sqrt{(1-c)(1-r)(1-c+r)}+2-2 c+c r}{r(2-c)}\right],
\end{aligned}
$$

in which $r=R_{t} / R_{b}$ and $c=r E_{c} /\left(Q+E_{v}+E^{*}\right)$. When the angular momentum $l=0$, one has $c=1$ and the above equations take a simpler form. The quantity $m$ used in some previous publications is expressed simply as $m=1-c$.

In order to reduce the number of fitting parameters, we took $E_{v}=E_{\text {cor }}$, although it is evident that, owing to the exponential dependence, any small variation of $E_{\text {cor }}$ induces a large change of $T$, and thus plays a more important role compared to the preexponential factor variation due to $E_{v}$ [see Eq. (1)]. Both shell and pairing effects are included in $E_{\text {cor }}=a_{i}\left(A_{e}\right) Q(i=1,2,3,4$ for even-even, odd-even, even-odd, and odd-odd parent nuclei). For a given cluster radioactivity we have four values of the coefficients $a_{i}$, the largest for the even-even parent and the smallest for the odd-odd one [8].

With only few exceptions, in the region of protonrich nuclei under investigation, measured $\alpha$-decay partial half-lives are not available. In principle, we can use the ASAFM to estimate these quantities. Nevertheless, slightly better results can be obtained by using our semiempirical formula

$$
\log _{10} T_{\alpha}=0.43429 K_{s} \chi-20.446
$$

where

$$
\begin{aligned}
K_{s}= & 2.52956 Z_{d a}\left[A_{d a} /\left(A Q_{\alpha}\right)\right]^{1 / 2} \\
& \times[\arccos \sqrt{x}-\sqrt{x(1-x)}], \\
x= & 0.423 Q_{\alpha}\left(1.5874+A_{d a}^{1 / 3}\right) / Z_{d a},
\end{aligned}
$$
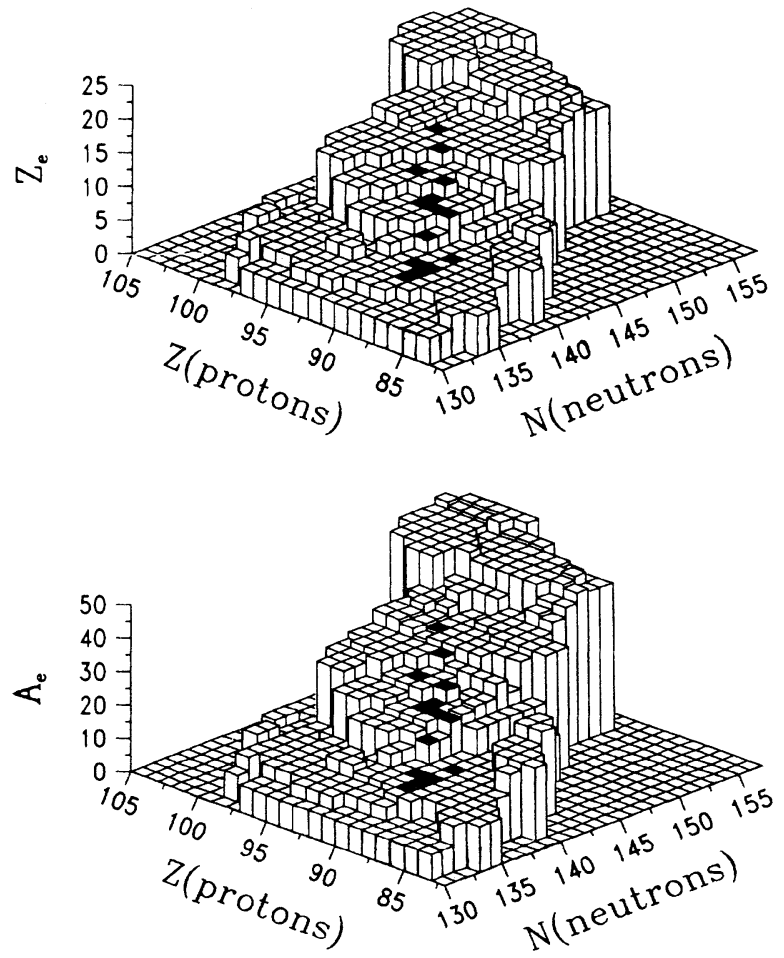

FIG. 1. Atomic and mass numbers of the most probable emitted clusters from trans-lead nuclei, predicted within the ASAFM. Fourteen experimental confirmations are marked with black on top. 


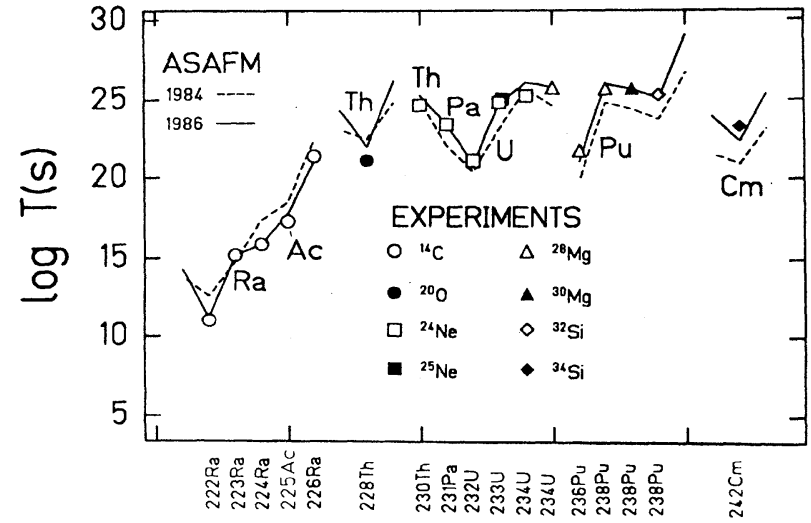

FIG. 2. Comparison of the early predictions (dashed lines) and those made by taking into account pairing forces (solid lines) with experimental points. In ${ }^{233} \mathrm{U}$ and ${ }^{238} \mathrm{Pu}$ it was not possible to resolve ${ }^{24} \mathrm{Ne}$ from ${ }^{25} \mathrm{Ne}$ and ${ }^{28} \mathrm{Mg}$ from ${ }^{30} \mathrm{Mg}$.

and the numerical coefficient $\chi$, close to unity, is a secondorder polynomial

$$
\chi=B_{1}+B_{2} y+B_{3} z+B_{4} y^{2}+B_{5} y z+B_{6} z^{2}
$$

in the reduced variables $y$ and $z$, expressing the distance from the closest magic-plus-one neutron and proton num- bers $N_{i}$ and $Z_{i}$ :

$$
\begin{aligned}
& y \equiv\left(N-N_{i}\right) /\left(N_{i+1}-N_{i}\right), N_{i}<N \leq N_{i+1}, \\
& z \equiv\left(Z-Z_{i}\right) /\left(Z_{i+1}-Z_{i}\right), Z_{i}<Z \leq Z_{i+1},
\end{aligned}
$$

with $N_{i}=\ldots, 51,83,127,185, \ldots, Z_{i}=\ldots, 29,51$, $83,115, \ldots$, and $Z_{d a}=Z-2, A_{d a}=A-4$. The coefficients $B_{i}$ are given in the second volume of Ref. [2].

The predicted, most probable emitted clusters from parent nuclei with $Z=82-104$ and $N=130-157$ are plotted in Fig. 1. Both the atomic numbers (upper part) and the mass numbers (lower part) are shown to be confirmed by experiments (black top).

A quantitative comparison is made in Fig. 2, where the measured half-lives are represented by points and our predictions of 1984 and 1986 (after taking properly into account the even-odd effect) by lines. As one can see, generally within one order of magnitude, the agreement is good; hence, we are confident in our estimations, provided the masses $M, M_{e}, M_{d}$ determining the released energy

$$
Q=\left(M-M_{e}-M_{d}\right) c^{2}
$$

are accurately known. Any erroneous increasement of the $Q$ value is reflected in a lower $K$, producing a drastic decrease of the half-life, because of the exponential de-

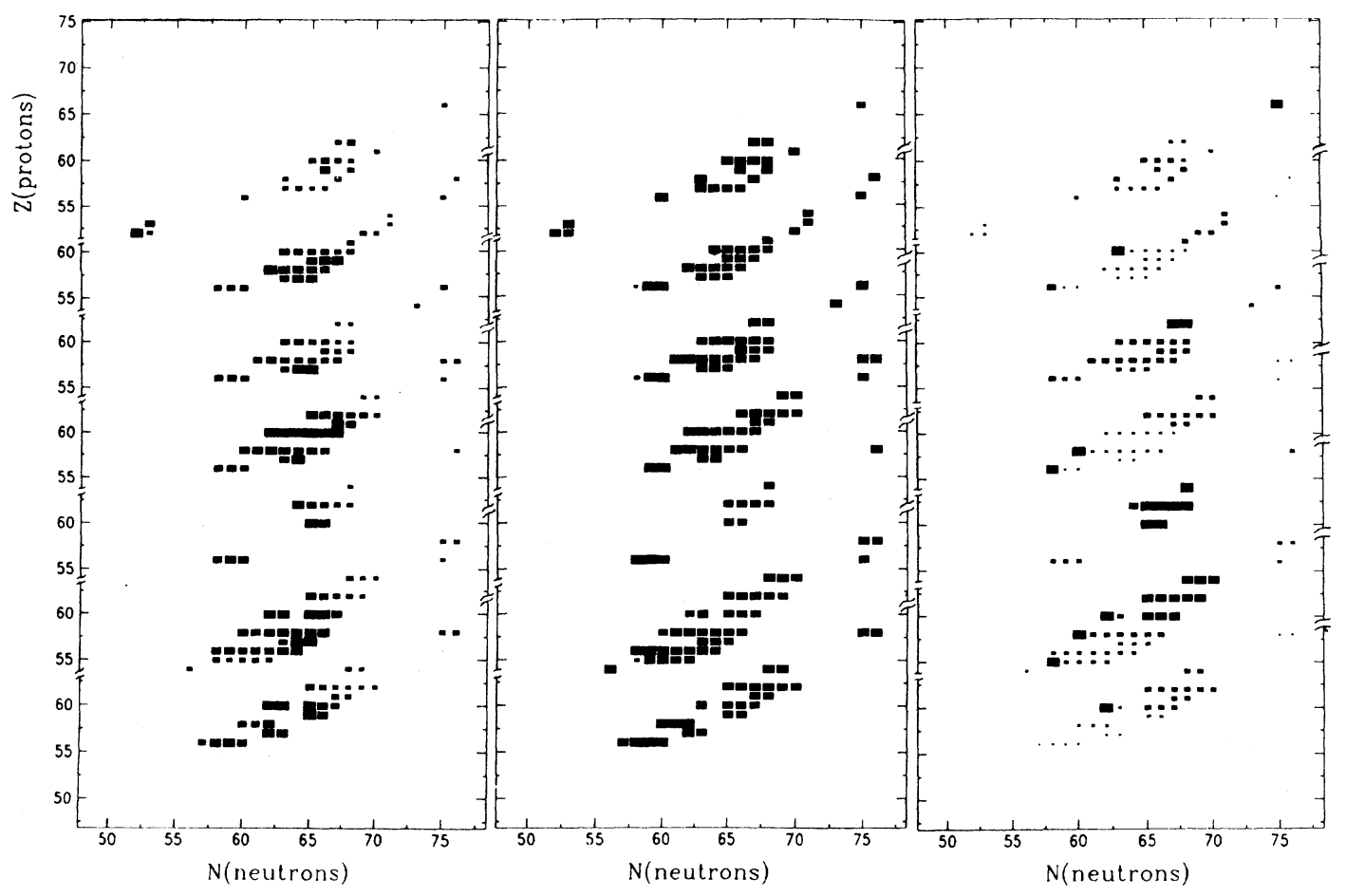

FIG. 3. "Cluster" representation of branching ratios $b$ with respect to $\alpha$ decay (left-hand side), parent nuclei half-lives $T$ (middle), and atomic numbers of emitted clusters $Z_{e}$ (right-hand side). For each parent nucleus, with $N$ neutrons and $Z$ protons, only the most probable emitted cluster is selected, according to the calculations based on seven mass tables. The seven islands, shown on each of the three parts of the figure, in descending order from top to the bottom, correspond to the following mass codes: $C=6,8,2,5,4,3$, and 7 . The dimension of each small square, with a given $N$ and $Z$, is carrying the information about the magnitude of the plotted quantity: A small (light) point represent a low value of $b$ on the left-hand figure, a long half-life $T$ on the central figure, and a low value of $Z_{e}$ on the right-hand figure. On the contrary, a large (solid) square means either a large $b$, a short $T$, or a high value of $Z_{e}$, respectively. 


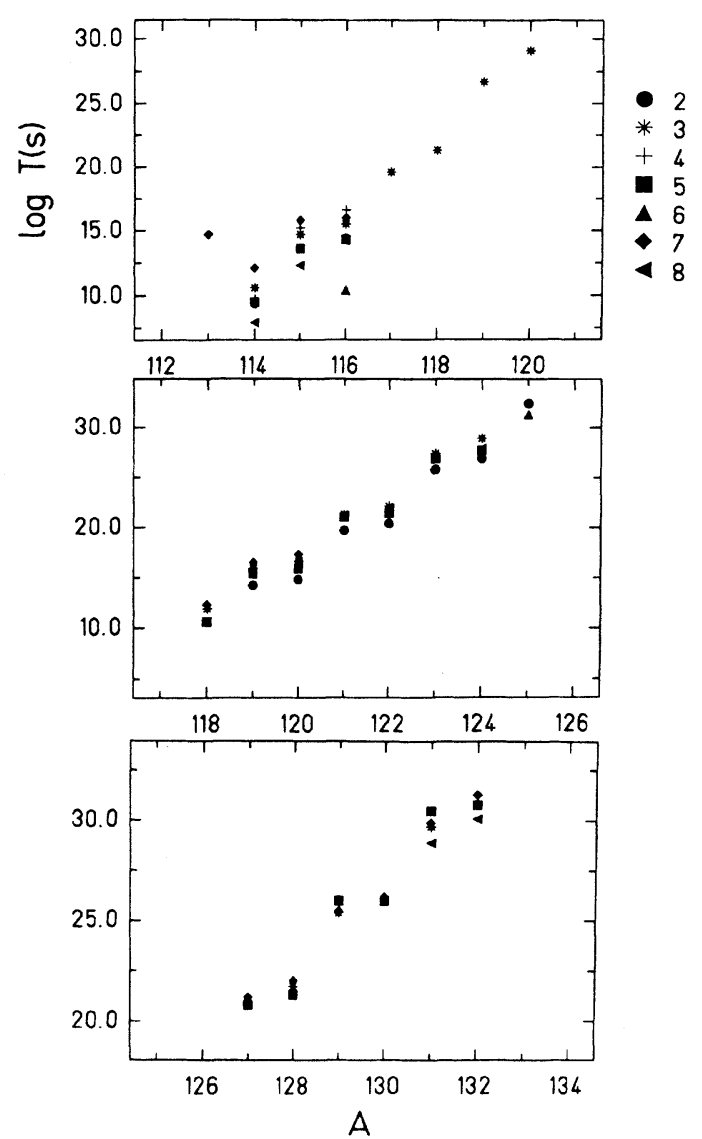

FIG. 4. Half-lives versus atomic numbers of parent nuclei for ${ }^{12} \mathrm{C}$ decay of $\mathrm{Ba}$ isotopes (top), ${ }^{16} \mathrm{O}$ radioactivity of $\mathrm{Ce}$ isotopes (intermediate), and ${ }^{28} \mathrm{Si}$ emission from $\mathrm{Sm}$ isotopes (bottom). The legend of the input mass codes is given in the upper part. Not all mass codes have been considered for $\mathrm{Ce}$ and $\mathrm{Sm}$, in order to illustrate better the systematic trend.

pendence shown in Eq. (1). Consequently, we need mass values as close as possible to the true ones.

Whenever available, we use the measured masses (mass code $C=0)$ or those determined from systematics $(C=$ 1) by Wapstra, Audi, and Hoekstra [11]. Otherwise, we try the mass estimations [12] one after the other, and then we compare the results. The following mass codes are conventionally adopted: $C=3$ for the estimations of Jänecke and Masson [13], $C=2$ for Masson and Jänecke [14], $C=4$ for Spanier and Johansson [15], $C=5$ for Tachibana et al. [16], $C=6$ for Satpathy and Nayak [17], $C=7$ for Comay, Kelson, and Zidon [18], and $C=8$ for Möller et al. [19].

In fact, we made calculations for seven input mass tables. The first one, possessing a central region around the line of beta stability where $C=0$ or $C=1$, is bordered on the neutron-rich and proton-rich sides by $C=3$ masses. For a general discussion we shall say that this is the $C=3$ region, in spite of the fact that the core belongs to 0 and 1 . In all other six input mass tables we shall only consider the regions beyond the core with "pure" $C=2,4,5,6,7,8$, respectively.

We have performed a large number of systematic calculations (25685 input masses times about 200 possible emitted clusters). From the results we usually selected those which have $T<10^{25} \mathrm{yr}$ and branching ratio relative to $\alpha$ decay $b=T_{\alpha} / T>10^{-18}$.

\section{RESULTS}

Theoretically, if no restriction is imposed to half-lives $T$ and branching ratios $b$, the region of cluster emitters is broader than that of $\alpha$ emitters [20]. As a general trend, light clusters are preferentially emitted from neutrondeficient nuclei and the heavy ones from neutron-rich parents.

The cluster kinetic energy per nucleon $E_{k}=$ $Q A_{d} /\left(A A_{e}\right)$ increases toward heavier proton-rich parents. Some shell effects are also visible not far from the magic numbers of nucleons 82 and 126 .

The half-life is more sensitive to the shell closure. Short half-lives appear not only in three regions of the proton rich nuclei, due to the daughter neutron magic numbers 50,82 , and 126 , where $\alpha$ decay is in competition, but also for $\alpha$-stable neutron-rich parents emitting neutron-rich clusters around ${ }_{28}^{78} \mathrm{Ni}_{50}$.

In practice, with presently available experimental techniques, the phenomena of cluster decays can only be detected when $T$ is low enough and $b$ is sufficiently high. $\mathrm{Up}$ to now the longest measured lifetime is of the order of $10^{26} \mathrm{~s}$ and the lowest measured branching ratio is almost $10^{-17}$.

By selecting $T<10^{30} \mathrm{~s}$ and $b>10^{-18}$, the area of cluster emitter regions became considerably smaller.

Besides the main region of trans-radium parents, where experiments have been done, another one with $Z=56-64$, $N=58-72$ looks very promising. Much higher branching ratios than the highest $\left(10^{-9}\right)$ measured until now are expected to be found in this island. Its real extension can be seen in Fig. 3, where the branching ratios, the half-lives, and the atomic numbers of the most probable emitted clusters from each parent nucleus are plotted. Despite the differences [some time even of qualitative nature, such as, for example, various most probable emitted clusters (see the right side of Fig. 3)] from table to table, at first sight they look similar. The even-odd effect, especially clear for protons, is present in all cases; some of the odd- $Z$ nuclides give no contribution fulfilling the above mentioned conditions.

For lighter neutron-deficient isotopes, when the "distance" from the line of beta stability increases, the halflives become shorter and shorter, as can be better seen on the selected examples plotted in Fig. 4, where the even-odd staggering appears as a staircase shape.

In the upper part of Fig. 4, for the $\mathrm{Ba}$ isotopes with $A=117-120$, the mass code is in fact $C=1$ (see Table I); hence, we only took into consideration the masses of Wapstra, Audi, and Hoekstra [11]. The other mass tables are coming into play for $A \leq 116$. Some of them (such as, for example, $C=7$ at $\bar{A}=113$ and $C=6$ at $A=$ 
TABLE I. ${ }^{12} \mathrm{C}$ emission from some proton-rich $\mathrm{Ba}$ and $\mathrm{La}$ isotopes.

\begin{tabular}{|c|c|c|c|c|c|c|c|}
\hline$Z$ & $A$ & $C$ & $C_{d}$ & $C_{d \alpha}$ & $\begin{array}{c}E_{k} / A_{e} \\
(\mathrm{MeV} / \text { nucleon })\end{array}$ & $-\log _{10} b$ & $\log _{10} T(s)$ \\
\hline \multirow[t]{6}{*}{$\overline{56}$} & 114 & 8 & 1 & 1 & 1.50 & 11.0 & 7.9 \\
\hline & & 2 & 1 & 1 & 1.45 & 9.3 & 9.4 \\
\hline & & 5 & 1 & 1 & 1.44 & 9.1 & 9.5 \\
\hline & & 4 & 1 & 1 & 1.44 & 8.8 & 9.7 \\
\hline & & 3 & 1 & 1 & 1.40 & 7.2 & 10.6 \\
\hline & & 7 & 1 & 1 & 1.36 & 4.0 & 12.1 \\
\hline \multirow[t]{6}{*}{56} & 115 & 8 & 1 & 1 & 1.40 & 11.0 & 12.3 \\
\hline & & 2 & 1 & 1 & 1.36 & 8.8 & 13.6 \\
\hline & & 5 & 1 & 1 & 1.36 & 8.9 & 13.6 \\
\hline & & 3 & 1 & 1 & 1.33 & 6.4 & 14.7 \\
\hline & & 4 & 1 & 1 & 1.31 & 5.0 & 15.2 \\
\hline & & 7 & 1 & 1 & 1.30 & 3.2 & 15.8 \\
\hline \multirow[t]{7}{*}{56} & 116 & 6 & 1 & 1 & 1.41 & 14.6 & 10.4 \\
\hline & & 8 & 1 & 1 & 1.30 & 10.5 & 14.3 \\
\hline & & 5 & 1 & 1 & 1.30 & 10.5 & 14.3 \\
\hline & & 2 & 1 & 1 & 1.30 & 10.4 & 14.4 \\
\hline & & 3 & 1 & 1 & 1.26 & 8.3 & 15.5 \\
\hline & & 7 & 1 & 1 & 1.25 & 7.2 & 16.0 \\
\hline & & 4 & 1 & 1 & 1.24 & 5.9 & 16.6 \\
\hline 56 & 117 & 1 & 0 & 0 & 1.20 & 7.6 & 19.6 \\
\hline 56 & 118 & 1 & 0 & 1 & 1.13 & 8.4 & 21.3 \\
\hline 56 & 119 & 1 & 1 & 1 & 1.05 & 4.1 & 26.7 \\
\hline 56 & 120 & 1 & 0 & 1 & 0.97 & 3.1 & 29.1 \\
\hline \multirow[t]{6}{*}{57} & 120 & 6 & 1 & 0 & 1.20 & 16.5 & 21.2 \\
\hline & & 3 & 1 & 0 & 1.14 & 11.5 & 24.1 \\
\hline & & 2 & 1 & 0 & 1.13 & 11.3 & 24.2 \\
\hline & & 8 & 1 & 0 & 1.12 & 9.5 & 25.0 \\
\hline & & 5 & 1 & 0 & 1.12 & 9.0 & 25.1 \\
\hline & & 7 & 1 & 0 & 1.09 & 4.6 & 26.5 \\
\hline \multirow[t]{6}{*}{57} & 121 & 6 & 0 & 0 & 1.16 & 14.6 & 21.8 \\
\hline & & 8 & 0 & 0 & 1.07 & 4.3 & 26.0 \\
\hline & & 2 & 0 & 0 & 1.06 & 3.2 & 26.3 \\
\hline & & 3 & 0 & 0 & 1.06 & 2.4 & 26.5 \\
\hline & & 5 & 0 & 0 & 1.06 & 1.9 & 26.7 \\
\hline & & 7 & 0 & 0 & 1.01 & -15.0 & 29.3 \\
\hline
\end{tabular}

TABLE II. ${ }^{16} \mathrm{O}$ radioactivity of some proton-rich $\mathrm{Ce}$ and $\mathrm{Nd}$ isotopes.

\begin{tabular}{|c|c|c|c|c|c|c|c|}
\hline $\bar{Z}$ & $A$ & $C$ & $C_{d}$ & $C_{d \alpha}$ & $\begin{array}{c}E_{k} / A_{e} \\
(\mathrm{MeV} / \text { nucleon })\end{array}$ & $-\log _{10} b$ & $\log _{10} T(s)$ \\
\hline \multirow[t]{5}{*}{58} & 118 & 5 & 1 & 5 & 1.61 & 5.6 & 10.6 \\
\hline & & 8 & 1 & 8 & 1.61 & -2.7 & 11.0 \\
\hline & & 3 & 1 & 3 & 1.58 & 5.2 & 11.9 \\
\hline & & 7 & 1 & 7 & 1.57 & 8.5 & 12.3 \\
\hline & & 4 & 1 & 4 & 1.51 & -16.7 & 14.5 \\
\hline \multirow[t]{6}{*}{58} & 120 & 2 & 1 & 2 & 1.50 & 7.9 & 14.8 \\
\hline & & 5 & 1 & 5 & 1.47 & 3.6 & 15.9 \\
\hline & & 8 & 1 & 8 & 1.45 & 0.2 & 16.6 \\
\hline & & 3 & 1 & 3 & 1.45 & 5.5 & 16.6 \\
\hline & & 7 & 1 & 7 & 1.43 & 4.7 & 17.3 \\
\hline & & 4 & 1 & 4 & 1.36 & -18.4 & 20.8 \\
\hline \multirow[t]{6}{*}{58} & 121 & 6 & 0 & 1 & 1.48 & 16.7 & 16.9 \\
\hline & & 2 & 0 & 1 & 1.41 & 10.3 & 19.7 \\
\hline & & 5 & 0 & 1 & 1.38 & 5.5 & 21.0 \\
\hline & & 8 & 0 & 1 & 1.38 & 4.4 & 21.2 \\
\hline & & 3 & 0 & 1 & 1.38 & 4.0 & 21.3 \\
\hline & & 7 & 0 & 1 & 1.36 & -0.9 & 22.2 \\
\hline 58 & 122 & 2 & 0 & 1 & 1.36 & 10.8 & 20.4 \\
\hline
\end{tabular}


TABLE II. (Continued).

\begin{tabular}{|c|c|c|c|c|c|c|c|}
\hline$Z$ & $A$ & $C$ & $C_{d}$ & $C_{d \alpha}$ & $\begin{array}{c}E_{k} / A_{e} \\
(\mathrm{MeV} / \text { nucleon }) \\
\end{array}$ & $-\log _{10} b$ & $\log _{10} T(s)$ \\
\hline & & 5 & 0 & 1 & 1.34 & 7.5 & 21.4 \\
\hline & & 3 & 0 & 1 & 1.32 & 4.4 & 22.1 \\
\hline & & 8 & 0 & 1 & 1.33 & 5.3 & 21.9 \\
\hline & & 7 & 0 & 1 & 1.30 & -2.9 & 23.3 \\
\hline \multirow[t]{5}{*}{58} & 123 & 2 & 1 & 1 & 1.28 & 11.1 & 25.8 \\
\hline & & 5 & 1 & 1 & 1.26 & 6.4 & 26.9 \\
\hline & & 8 & 1 & 1 & 1.26 & 6.6 & 26.9 \\
\hline & & 3 & 1 & 1 & 1.25 & 3.8 & 27.4 \\
\hline & & 7 & 0 & 1 & 1.23 & -2.2 & 28.3 \\
\hline \multirow[t]{5}{*}{58} & 124 & 2 & 0 & 1 & 1.22 & 12.1 & 26.9 \\
\hline & & 5 & 0 & 1 & 1.21 & 9.0 & 27.7 \\
\hline & & 8 & 0 & 1 & 1.20 & 7.8 & 27.9 \\
\hline & & 3 & 0 & 1 & 1.19 & 2.6 & 28.9 \\
\hline & & 7 & 0 & 1 & 1.17 & -4.2 & 29.8 \\
\hline \multirow[t]{4}{*}{60} & 122 & 5 & 1 & 5 & 1.52 & 2.0 & 16.7 \\
\hline & & 3 & 1 & 3 & 1.49 & 3.8 & 18.2 \\
\hline & & 7 & 1 & 7 & 1.47 & 1.7 & 19.1 \\
\hline & & 4 & 1 & 4 & 1.38 & -4.1 & 23.0 \\
\hline \multirow[t]{6}{*}{60} & 123 & 2 & 1 & 2 & 1.52 & 9.3 & 18.6 \\
\hline & & 8 & 1 & 8 & 1.46 & 7.0 & 20.9 \\
\hline & & 5 & 1 & 5 & 1.44 & 2.2 & 22.0 \\
\hline & & 3 & 1 & 3 & 1.42 & 3.0 & 22.6 \\
\hline & & 7 & 1 & 7 & 1.39 & 0.9 & 23.8 \\
\hline & & 4 & 1 & 4 & 1.38 & -0.6 & 28.3 \\
\hline
\end{tabular}

116), being out of the general trend, could be, very likely, ignored. In the next two plots, concerning $\mathrm{Ce}$ and $\mathrm{Sm}$ isotopes, we have made a selection, by removing some points, in order to illustrate better the normal trend. A complete list of results for some isotopes of $\mathrm{Ba}, \mathrm{La}$, $\mathrm{Ce}, \mathrm{Nd}$, and $\mathrm{Sm}$ is presented in Tables I-III, where the cluster kinetic energies per nucleon and the mass codes of the daughter $C_{d}$, and of the $\alpha$-decay daughter $C_{d \alpha}$, are also given. Other emitted clusters may be found in Ref. $[8]$.
In some of the calculation results presented in Table II, the branching ratio is larger than unity. This overoptimistic behavior could be a consequence of a too pesimistic estimation of the $\alpha$-decay $Q_{\alpha}$ value, due to errors in the masses of the parent and $\alpha$-decay daughter, for $C=C_{d \alpha}=4,7,8, C_{d}=1$, or a too optimistic clusterdecay $Q$ value, for $C_{d}=0,1, C_{d \alpha}=1$. At the same time we have no special reason to exclude a priori the possibility of the occurrence of such very enhanced cluster decays in competition with $\alpha$ emission, as long as a whole

TABLE III. ${ }^{28} \mathrm{Si}$ emission from some neutron-deficient $\mathrm{Nd}$ and $\mathrm{Sm}$ isotopes.

\begin{tabular}{|c|c|c|c|c|c|c|c|}
\hline$Z$ & $A$ & $C$ & $C_{d}$ & $C_{d \alpha}$ & $\begin{array}{c}E_{k} / A_{e} \\
(\mathrm{MeV} / \text { nucleon })\end{array}$ & $-\log _{10} b$ & $\log _{10} T(s)$ \\
\hline \multirow[t]{7}{*}{$\overline{60}$} & 125 & 6 & 0 & 6 & 1.51 & 13.3 & 18.8 \\
\hline & & 2 & 0 & 2 & 1.40 & 12.1 & 23.3 \\
\hline & & 8 & 0 & 8 & 1.43 & 10.6 & 25.2 \\
\hline & & 5 & 0 & 5 & 1.42 & 1.3 & 26.1 \\
\hline & & 3 & 0 & 3 & 1.41 & 1.2 & 26.4 \\
\hline & & 7 & 0 & 7 & 1.40 & 2.0 & 27.0 \\
\hline & & 4 & 0 & 4 & 1.35 & 0.9 & 30.3 \\
\hline \multirow[t]{4}{*}{62} & 127 & 5 & 1 & 5 & 1.59 & 3.3 & 20.8 \\
\hline & & 3 & 1 & 3 & 1.59 & 6.1 & 20.9 \\
\hline & & 7 & 1 & 7 & 1.59 & 9.5 & 21.2 \\
\hline & & 4 & 1 & 4 & 1.52 & 7.4 & 24.9 \\
\hline \multirow[t]{4}{*}{62} & 128 & 5 & 1 & 5 & 1.56 & 5.0 & 21.3 \\
\hline & & 3 & 1 & 3 & 1.55 & 8.8 & 21.7 \\
\hline & & 7 & 1 & 7 & 1.55 & 12.7 & 22.0 \\
\hline & & 4 & 1 & 4 & 1.49 & 9.6 & 25.4 \\
\hline
\end{tabular}



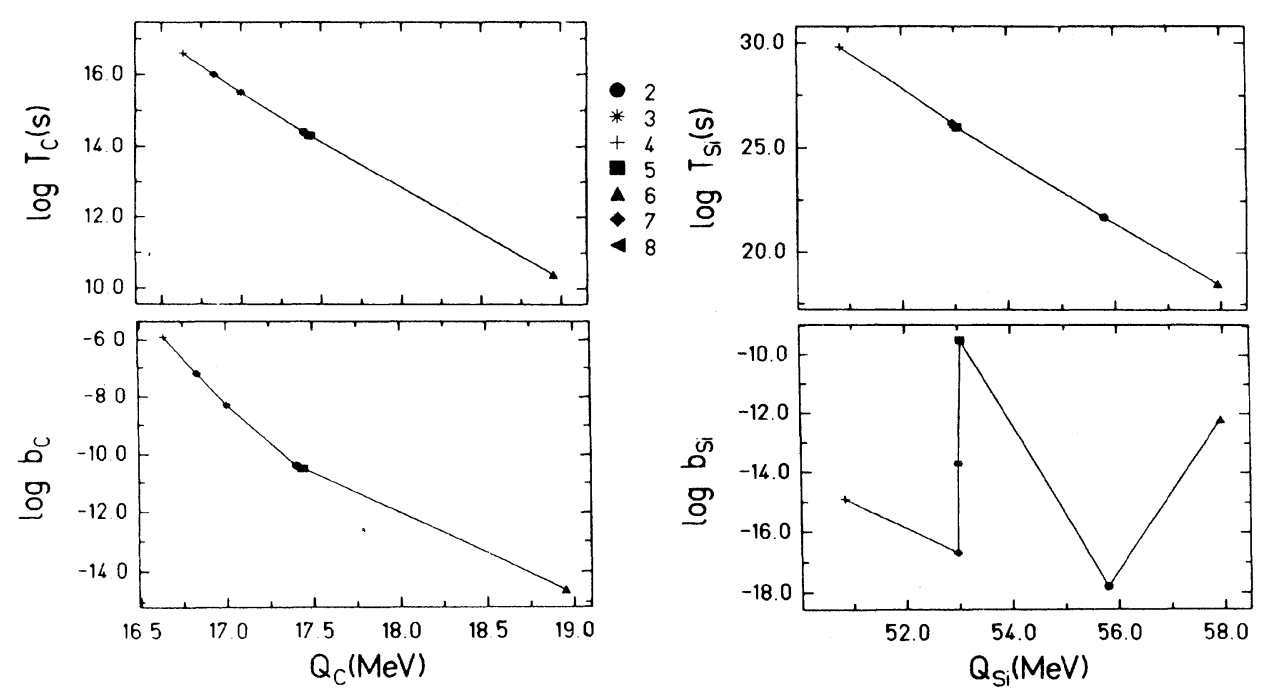

FIG. 5. Variation of half-life and branching ratio for ${ }^{12} \mathrm{C}$ decay of ${ }^{116} \mathrm{Ba}\left(C_{d}=C_{d \alpha}=1\right)$ and for ${ }^{28} \mathrm{Si}$ radioactivity of ${ }^{130} \mathrm{Sm}$ $\left(C_{d}=1, C_{d \alpha}=C\right)$ with the $Q$ value calculated by using the input mass tables with the codes $C=2-8$.

island of cluster emission exists in a region with $Q_{\alpha}<0$. Also, for ${ }^{122,123} \mathrm{Nd}$, according to the calculations based on the mass table with $C=4$, the most probable emitted cluster should be ${ }^{24} \mathrm{Mg}$ instead of ${ }^{16} \mathrm{O}$.

Again, in a similar way to what happened in the transradium part of the nuclear chart, the branching ratio law of variation (Fig. 3) does not follow that of the half-life. Usually the maxima are placed around the center of the island, and not on the "west-side shore."

The sensitivity of the estimated half-life and branching ratio to the uncertainty in the mass value of parent nucleus (left-hand side of Fig. 5) and that of both parent nucleus and $\alpha$-daughter (right-hand side of Fig. 5) is relatively high: about $(6.2$ orders of magnitude $) /(2.35 \mathrm{MeV})$ for $T$ and (8.7 orders) $/(2.35 \mathrm{MeV})$ for $b$ in the former case and (11.3 orders)/(7.1 MeV) for $T$ in the latter.

In conclusion, despite the large uncertainty in the estimated mass values of nuclei far from stability, we believe that there is a real chance to detect ${ }^{12} \mathrm{C},{ }^{16} \mathrm{O},{ }^{28} \mathrm{Si}$ radioactivities (and may be others) of some neutrondeficient nuclides with $Z=56-64$ and $N=58-72$. Let us consider, as an example, the ${ }^{12} \mathrm{C}$ radioactivity of ${ }^{114} \mathrm{Ba}$, which we mentioned as interesting few years ago. If we take into account the estimated [21] $\beta$-decay half-life of $0.3-0.5 \mathrm{~s}$, the branching ratio with respect to this de- cay mode could be in the range $10^{-8}-10^{-12} \mathrm{~s}$, which is measurable in an on-line experiment. In fact, branching ratios with respect to $\alpha$ decay as low as $10^{-16}$ have been already experimentally observed. In a recently performed measurement, the exotic nucleus ${ }^{114} \mathrm{Ba}$ was produced by bombarding a Ni target with $280 \mathrm{MeV}{ }^{58} \mathrm{Ni}$ heavy ions. According to these preliminary results from Dubna [22], about 10 events (of ${ }^{12} \mathrm{C}$ ) have been observed. There are difficulties connected with the high background produced by neutrons. In a new experiment to be done at GSI Darmstadt [23], one can improve considerably the experimental conditions, by transporting the reaction products far from the region with a strong neutron background. Reaction cross section $\beta, \alpha$, and $\mathrm{C}$ decays are planned to be determined. Radioactive beams could be alternatively used to produce nuclei far from stability which are cluster emitters. From the measured kinetic energy of the emitted cluster and of the $\alpha$ and $\beta$ particles, we can get improved values of the implied masses, which in turn could be used to obtain more reliable estimates of halflives and branching ratios.

This work was supported by Bundesministerium für Forschung und Technologie, by Gesellschaft für Schwerionenforschung, and by Institute of Atomic Physics.
[1] W. Greiner, M. Ivaşcu, D. N. Poenaru, and A. Săndulescu, in Treatise on Heavy Ion Science, edited by D. A. Bromley (Plenum, New York, 1989), Vol. 8, p. 641.

[2] D. N. Poenaru, M. Ivaşcu, and W. Greiner, in Particle Emission from Nuclei, edited by D. N. Poenaru and M. Ivaşcu (CRC, Boca Raton, FL, 1989), Vol. III, p. 203.

[3] D. N. Poenaru and W. Greiner, in Proceedings of the International Conference on Clustering Phenomena in Atoms and Nuclei, Turku, Finland, 1991, edited by M. Brenner, T. Lönnroth, and B. Malik (Springer, Heidel- berg, 1992), p. 235

[4] P. B. Price, Annu. Rev. Nucl. Part. Sci. 39, 19 (1989).

[5] E. Hourani, M. Hussonnois, and D. N. Poenaru, Ann. Phys. (Paris) 14, 311 (1989).

[6] R. Bonetti, C. Chiesa, A. Guglielmetti, C. Migliorino, A. Cesana, and M. Terrani, in Proceedings of the International Conference on Nuclear Reaction Mechanisms, Varenna, edited by E. Gadioli (Ricerca Scientifica ed Educazione Permanente, Milano, 1991), Suppl. No. 84, p. 333. 
[7] S. P. Tretyakova, V. L. Mikheev, Yu. S. Zamyatnin, and A. A. Ogloblin, in Proceedings of the International Conference on Exotic Nuclei, Foros, Ukraina, 1991, edited by Yu. E. Penionzhkevich and R. Kalpakchieva (World Scientific, Singapore, in press).

[8] D. N. Poenaru, D. Schnabel, W. Greiner, D. Mazilu, and R. Gherghescu, At. Data Nucl. Data Tables 48, 231 (1991).

[9] E. Roeckl and D. Schardt, in [2], Vol. II, p. 1.

[10] D. N. Poenaru, J. A. Maruhn, W. Greiner, M. Ivaşcu, D. Mazilu, and I. Cata, Z. Phys. A 333, 291 (1989).

[11] A. H. Wapstra, G. Audi, and R. Hoekstra, At. Data Nucl. Data Tables 39, 281 (1988).

[12] P. E. Haustein, At. Data Nucl. Data Tables 39, 185 (1988).

[13] J. Jänecke and P. J. Masson, At. Data Nucl. Data Tables 39, 265 (1988)

[14] P. J. Masson and J. Jänecke, At. Data Nucl. Data Tables 39, 273 (1988).
[15] L. Spanier and S. A. E. Johansson, At. Data Nucl. Data Tables 39, 259 (1988).

[16] T. Tachibana, M. Uno, M. Yamada, and S. Yamada, At. Data Nucl. Data Tables 39, 251 (1988).

[17] L. Satpathy and R. K. Nayak, At. Data Nucl. Data Tables 39, 241 (1988).

[18] E. Comay, I. Kelson, and A. Zidon, At. Data Nucl. Data Tables 39, 235 (1988).

[19] P. Möller, W. D. Myers, W. J. Swiatecki, and J. Treiner, At. Data Nucl. Data Tables 39, 225 (1988).

[20] D. N. Poenaru, W. Greiner, M. Ivaşcu, and A. Săndulescu, Phys. Rev. C 32, 2198 (1985).

[21] M. Hirsch, A. Staudt, K. Muto, and H. V. KlapdorKleingrothaus (unpublished).

[22] Yu. Ts. Oganessian, V. L. Mikheev, and S. P. Tretyakova (private communication).

[23] P. B. Price, E. Roeckl, and R. Bonetti (private communication). 